\title{
DISSIMILARITY MEASURE OF CONSECUTIVE FRAMES IN WIRELESS CAPSULE ENDOSCOPY VIDEOS: A WAY OF SEARCHING FOR ABNORMALITIES
}

\author{
ISABEL NARRA FIGUEIREDO, CARLOS LEAL, LUÍS PINTO, PEDRO NARRA \\ FIGUEIREDO AND RICHARD TSAI
}

\begin{abstract}
In a previous work we have shown that the curve representing the dissimilarity measure between consecutive frames of a wireless capsule endoscopic video of the small bowel, obtained by means of an image registration method, can be regarded as a rough indicator of the speed of the capsule, and simultaneously, it is also a valuable auxiliary medical tool. In effect, this curve enables a global and fast interpretation of the video, in the sense that it clearly divides the video frames into two main categories: consecutive frames with similar content, which correspond to low values in the curve, and consecutive frames displaying abrupt changes in the image content, which are depicted by peaks, i.e. high values, in the curve. As the main goal of a wireless capsule video examination consists in searching for abnormal features in the images, the purpose of the present work is to analyse whether this curve can also be used to search, quickly, for abnormalities. The experiments performed focus on bleeding identification in small bowel images.
\end{abstract}

KEYWORDS: elastic and affine image registration, multiscale representation, wireless capsule endoscopy.

AMS Subject Classification (2010): 68U10, 49M15, 65D05, 65D07, 65D18, 65K10, $65 \mathrm{M} 06$.

\section{Introduction}

Wireless capsule endoscopy (WCE) is a medical procedure used for the examination of the gastrointestinal tract (GI), see for example $[12,1,8,17$, $16,4]$. It consists of a miniature device, the capsule, that is ingested by a patient, and as it travels through the GI, moved by peristalsis (the capsule does not have any motor) it transmits images of the internal lumen of the GI organs, to a data recorder worn on a belt outside the patient's body. Afterwards, the analysis of the WCE's video is done by a skilled medical doctor, whose main objective is to search for possible abnormalities in the images. This analysis requires firstly, a very high level of attention not to

Received March 08, 2017.

The first three authors acknowledge support from CMUC (UID/MAT/00324/2013 funded by the Portuguese Government through FCT/MCTES and co-funded by the European Regional Development Fund through Partnership Agreement PT2020). 
fail to notice abnormalities that might appear only in a single frame or that might be very difficult to identify, and, secondly, a lot of time, because the capsule transmits thousands of images (for instance, PillCAM SB, a WCE for the small bowel, operates during approximately 8 hours and transmits images at a rate of two frames per second).

Therefore in these last years many authors have focused their research on auxiliary computer-assisted methods (see for instance $[6,22,23,2,24]$ ), to facilitate the video interpretation, to avoid the risk of missing abnormalities, to reduce the time needed for the analysis of the video, and to determine the exact location of the abnormality (when a lesion is identified in an image, its exact location in the organ is not known, because the position of the capsule is unkown).

In a previous work [10] we proposed a multiscale elastic image registration procedure, that includes an affine pre-image registration, for tracking the motion of the capsule, and an indicator of the WCE speed was also obtained. This particular strategy intends to take into account the combination of two types of movements: the movement of the capsule, which is rigid-like, and the nonrigid movement of the small intestine, which is an elastic organ, that bends and deforms due to peristalsis. The affine pre-registration is performed at a coarse scale, and corresponds to an affine alignment of the two images at a coarse scale, thus matching the most prominent and large features, and correcting the main distortions (rotation and scale), originated by the WCE movement. In the second step, and based on the result of the first step, a multiscale elastic registration is accomplished. This second step performs the multiscale elastic registration, and corrects the fine and local misalignments generated by the non-rigid movement of the gastrointestinal tract. Moreover, for improving the approach, we iterate it once more, i.e., we use the estimated deformation as a better initial condition for the model in order to improve our estimate. Finally, a dissimilarity measure is defined in order to quantify the accuracy of the proposed registration procedure, or equivalently, to quantify the degree of dissimilarity/similarity of the two registered images. Furthermore, this dissimilarity measure is also an indicator of the speed of the capsule. In effect, a very low value of this measure indicates that the image content does not change in the corresponding pair of consecutive frames, thus this means that the capsule is almost still or rotates/moves slowly, because it is capturing the same scene. On the other hand, a high value of this dissimilarity measure indicates abrupt scene changes in the two consecutive 
frames, therefore the image content is changing, and consequently the capsule should have moved, or the background has changed dramatically due to peristalsis.

In the present paper we use precisely the image registration approach and the dissimilarity measure employed in [10], to investigate whether the curve representing this dissimilarity measure is a good identifier of frames with lesions in the WCE video. We focus on the identification of frames with bleeding, in the small bowel. In the literature there are several papers reporting different methods for the detection of bleeding in WCE images, that essentially rely on the color and also texture properties of the bleeding regions, and among them we cite $[5,7,11,13,19,18,20,9]$, with different degrees of success. To the best of our knowledge this is the first time that an image registration approach is used to detect frames with bleeding in WCE images. For instance, references [7] and [20] report that the sensitivity and specificity of the red indicator, which is an application included in the software that is sold with the PillCAM capsule, are approximately $37 \%$ and $59 \%$, and [11] reports $21.5 \%$ and $41.8 \%$ for the same application, which are very low results. So any alternative technique, as the one we are proposing, could be interesting to be explored in real-life frameworks. On the other hand, for example, in [9], much better sensitivity and specifity results are obtained. However, the benefit of the present approach, proposed herein, would be that it also gives the user an estimation of the capsule's motion, which may be an important information for physicians for treatment. Our main motivation for using this technique relies on the visual discrepancy originated by the different intensity profile and localization of bleeding regions in normal mucosa, which is likely to be detected by image registration techniques. Moreover, the outcome of the proposed approach is an efficient graphical tool that permits a global and rapid interpretation of a WCE video, by identifying a set of critical frames that might be of relevant medical interest. The results of the experiments we have performed for identifying frames with bleeding, in WCE images of the small bowel, with the proposed image registration approach, are promising, and forecast its efficiency in real-life medical environments, as commented in the last section of the paper.

After this introduction, the next Section 2 briefly describes the methodology introduced in [10]. In Section 3, the experiments and results, obtained with the aforementioned approach, are reported. Finally in Section 4 we present some conclusions and extensions. 


\section{Description of the Dissimilarity Measure}

A concise description of the adopted approach, that includes the dissimilarity measure, is summarized next, and we refer the reader to [10] for the details.

Since we are searching for bleeding regions and it is known that these regions appear more contrasted in the a-channel of the CIE Lab color space (see [9] and [21]), we always perform the registration approach in this achannel.

Then, let $R$ and $T$ be the a-channels of two consecutive images, that is, the reference and template images respectively, to be compared. We recall that the goal of image registration (see $[15,14]$ ) is to find a geometric transformation $v$, such that the transformed template image, denoted by $T(v)$, becomes similar to the reference image $R$ (we point out that we regard the graphs of the images as deforming surfaces). The transformation $v$ is affine, in the case of affine image registration, or, a more general transformation, in the case of elastic image registration [3]. Moreover, as the proposed approach uses a multiscale representation of the data, hereafter we denote by $R_{\theta}$ and $T_{\theta}$, the approximation of $R$ and $T$, at a scale $\theta$, by a spline interpolation procedure (by increasing $\theta$, increasingly smooth approximations $R_{\theta}$ and $T_{\theta}$ of $R$ and $T$, respectively, are obtained). Let $i=0,1, \ldots, n$ be an index indicating $n+1$ fixed scales, then we perform the following three steps:

- Step 1 - Registration of the pair $(R, T)$ :

(1) Solve the affine image registration problem for the pair $\left(R_{\theta_{0}}, T_{\theta_{0}}\right)$ at the coarse scale $\theta_{0}$. Get the solution $\varphi_{0}$.

(2) Solve the elastic registration problem at the coarse scale $\theta_{0}$, with the pair $\left(R_{\theta_{0}}, T_{\theta_{0}}\right)$, by using $\varphi_{0}$ as initial guess for the GaussNewton method. Get the solution $u_{\theta_{0}}$.

(3) For $i=1, \ldots, n$, at each scale $\theta_{i}$, solve the elastic registration problem, by using, for the Gauss-Newton method, $u_{\theta_{i-1}}$ as the initial guess. Get the solution $u_{\theta_{i}}$.

(4) Let $\tilde{u}$ be the solution at the final scale, that is, $\tilde{u}=u_{\theta_{n}}$.

- Step 2 - Register the pair $(R, T(\tilde{u}))$ with the proposed approach (or equivalently, repeat Step 1 with $T$ replaced by $T(\tilde{u}))$. Get the final solution $u$. 


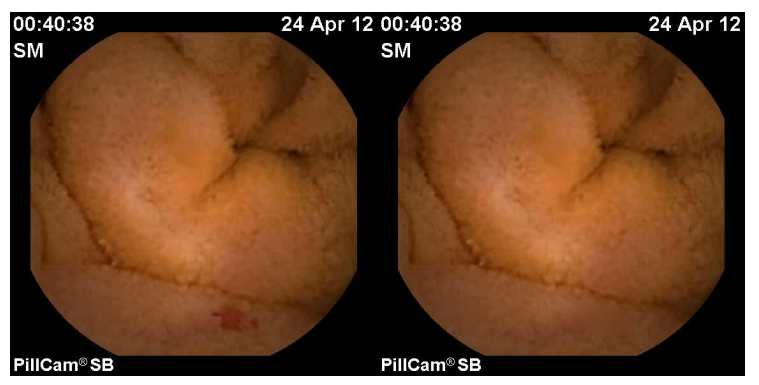

Figure 1. Test 1: On the left a frame displaying bleeding (which is the redish region appearing in the bottom right part of the image), and, on the right the same frame, without the bleeding region that was removed by using an in-painting technique.

- Step 3 - Compute the normalized dissimilarity measure $N D M$, defined by

$$
N D M:=\frac{\|T(u)-R\|_{L^{2}(\Omega)}}{\|R\|_{L^{2}(\Omega)}} .
$$

Here $L^{2}(\Omega)$ is the space of square-integrable functions in $\Omega$, a set that stands for the pixel domain, and $\|\cdot\|_{L^{2}(\Omega)}$ denotes the usual norm in $L^{2}(\Omega)$.

We remark that small bleeding regions, due to its drastic different intensity profile and localization, will not be accommodated by neither Step 1 nor Step 2, and thus will likely result in relatively large difference to the reference image.

\section{Tests and Results}

We have done three types of experiments with real WCE video frames, whose description and results are reported in this section. Test 1 is a synthetic experiment, Test 2 is a semi-synthetic experiment, and Test 3 is a real experiment.

In Test 1, we first consider a frame displaying bleeding, exhibited on the left of Figure 1, and the same frame without the bleeding region (displayed on the right of Figure 1), and that was removed by an in-painting technique. Then we generate 40 frames, where each one is an artificially modified version of this frame without bleeding. This modified version is obtained by applying a rotation (simulating the possible rotation of the capsule), a scale factor (simulating the shrinkage or expansion of the image and that depends on fact that the capsule is far away or approaches the imaged region), and an 

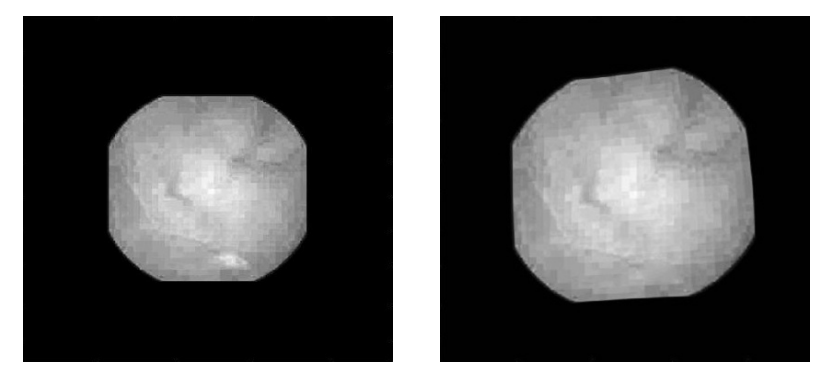

FiguRE 2. Test 1: a-channel images of the Reference (left) and Template (right), for pair number 8. The bleeding corresponds to the whitish region shown in the bottom right part of the Reference image.

elastic deformation (imitating the natural deformation of the intestine, due to peristaltic movement). Then we apply the image registration approach to this frame without bleeding and its rotated, scaled and elastic deformed versions, and compute the corresponding normalized dissimilarity measure NDM: there is a total of 40 pairs, but in four pairs (the pairs $8,10,22$ and 25) we use the original frame with bleeding. In Figure 2 we exhibit the a-channels images of pair number 8 , where the original frame (on the left) displays the bleeding region. The graphic of the $N D M$ curve is shown in Figure 3. As can be seen, this curve reaches peaks in the pairs 8, 10, 22 and 25 , which are precisely the pairs where one image has bleeding. Therefore the peaks of the dissimilarity measure $N D M$ clearly indicate the frames with bleeding.

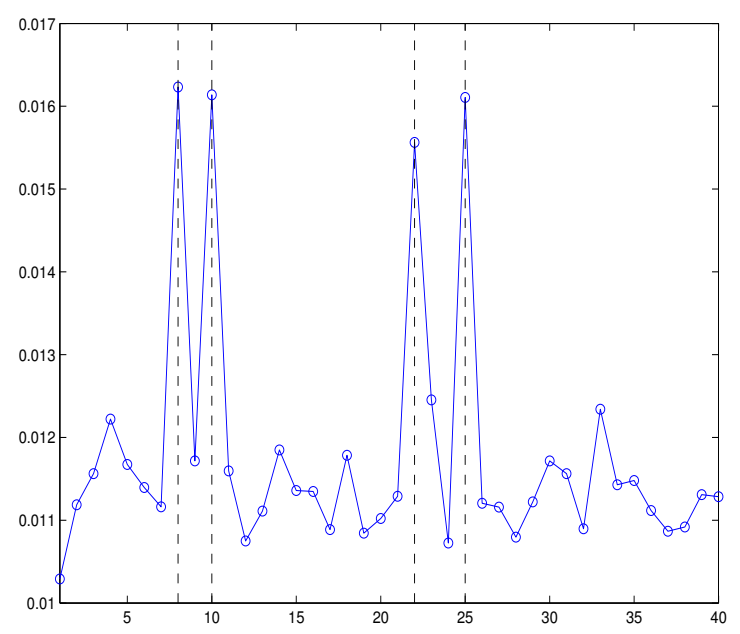

Figure 3. Test 1: NDM curve - in pairs 8, 10, 22 and 25 one image has a bleeding region. 

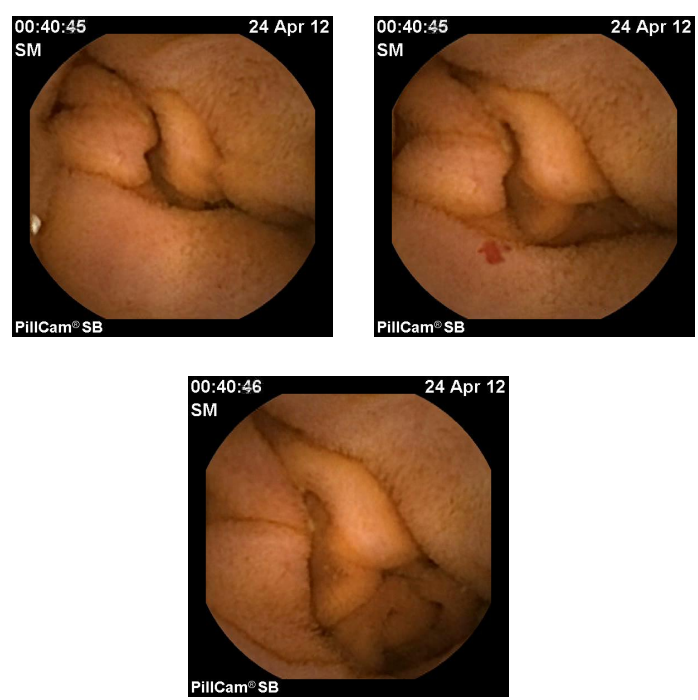

Figure 4. Test 2: Three consecutive frames. Top - frame 7 (left) and modified frame 8 with artificial bleeding (right). Bottom frame 9 .

In Test 2 we perform two semi-synthetic experiments. In the first experiment we consider a sequence with $23 \mathrm{WCE}$ video frames with no signs of bleeding. In frame 8 we artificially insert a bleeding region, where the inserted bleeding was a region removed from a real WCE frame displaying bleeding. Figure 4 shows frame 7 , the modified frame 8 with artificial bleeding and frame 9 . Then we apply the image registration approach to the consecutive frames (there is a total of 22 pairs), and compute the dissimilarity measure $N D M$ for the original WCE image sequence and the modified one (obtained by modifying frame number 8 with artificial bleeding). The two $N D M$ curves, that are shown in Figure 5, coincide as expected, except for the pairs 7 and 8, which correspond to the pairs containing the frames $(7,8)$ and $(8,9)$, respectively. Figure 5 clearly shows that the disparity between the two $N D M$ curves is generated precisely by the bleeding region in frame 8. The red $N D M$ curve, which represents the dissimilarity measure for the modified sequence, exhibits two large peaks in pairs 7 and 8, which correctly identify the single frame with bleeding in this sequence.

We have repeated this type of semi-synthetic experiment in another sequence of video frames, with 92 images displaying relatively clean mucosa without bleeding. We insert three variants of a real bleeding region, displaying different appearance in three images, with respect to color signature and size, and randomly put them in 8 frames and run the algorithm on both the 


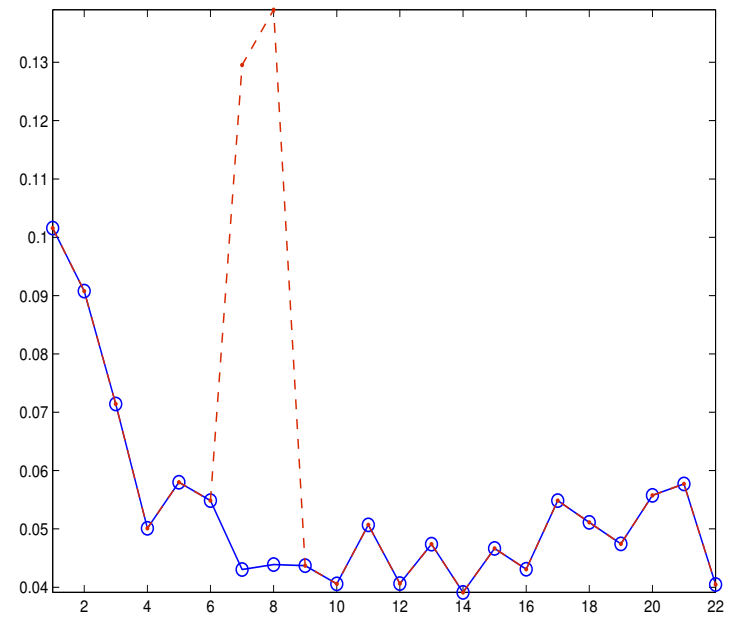

Figure 5. Test 2 (first experiment): $N D M$ curves, for the original sequence in blue, and for the modified sequence in red. In the red curve the pairs 7 and 8 contain the modified frame 8 with artificial bleeding.

original and modified sequences. The result is shown in Figure 6, where the blue curve is the $N D M$ of the original sequence and the red curve is the $N D M$ of the modified sequence. The two curves overlap except in 16 pairs

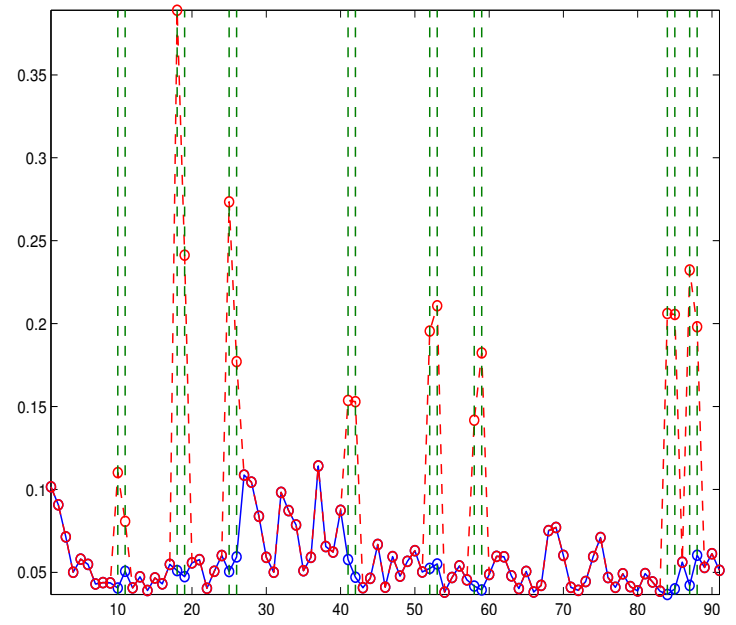

Figure 6 . Test 2 (second experiment): $N D M$ curves, for the original sequence in blue, and for the modified sequence (with artificial bleeding) in red. The 16 peaks in the red curve correspond to the 16 pairs with bleeding. 

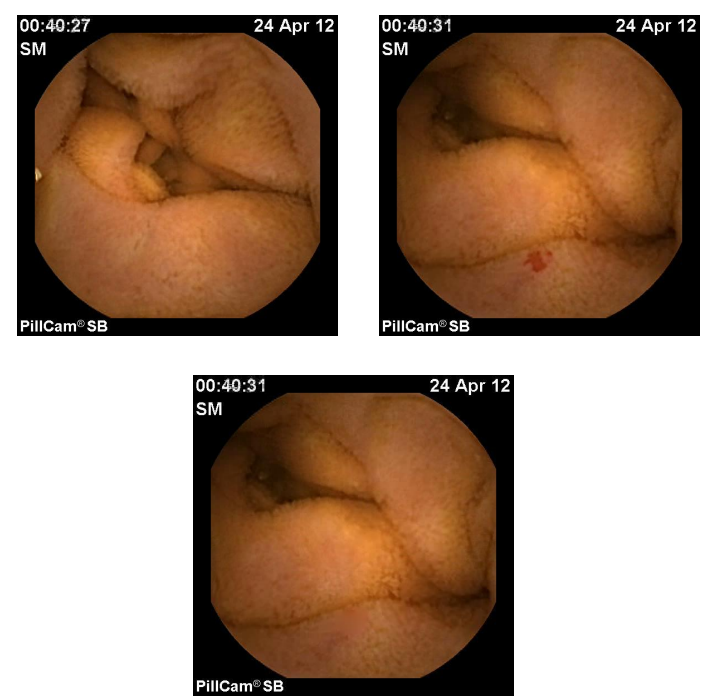

Figure 7. Test 3: Top - Consecutive frames 3 and 4, of pair number 3. Bottom - Modified frame 4 without the bleeding region (removed by an in-painting technique).

that have bleeding (there are 8 frames with bleeding that, consequently, originate 16 pairs with bleeding). Clearly the peaks in the red curve indicate the existence of bleeding.

Finally Test 3 is a real experiment. Here we take 32 consecutive frames in a WCE video, some exhibiting bleeding (has shown in Figure 7, in the top row), and build the corresponding $N D M$ curve by doing the image registration of the consecutive frames. This curve is shown in Figure 8, where a red circle indicates a pair where only one frame has bleeding, a green circle means a pair where both frames have bleeding and a black dot signifies that none of the frames in a pair has bleeding. The blue circle in pair number 3 is the value of $N D M$, when the bleeding region is removed from frame 4 (see Figure 7). As shown, this value is much lower than the $N D M$ value obtained with the original frame 4. This emphasis and demonstrates that the presence of bleeding can be detected with the dissimilarity measure used in this paper. Without bleeding the $N D M$ value is less than 0.08 and with bleeding the value more than doubles, it is over 0.18 . Thus, the highest $N D M$ value (in pair 3) again leads to the correct identification of a frame with bleeding. In pairs 4,5 and 6 the content of the images does not change significantly, as well as in pairs 13 to 31 , and this is the reason why the $N D M$ values for these pairs are relatively small. The $N D M$ values of the remainder pairs are 


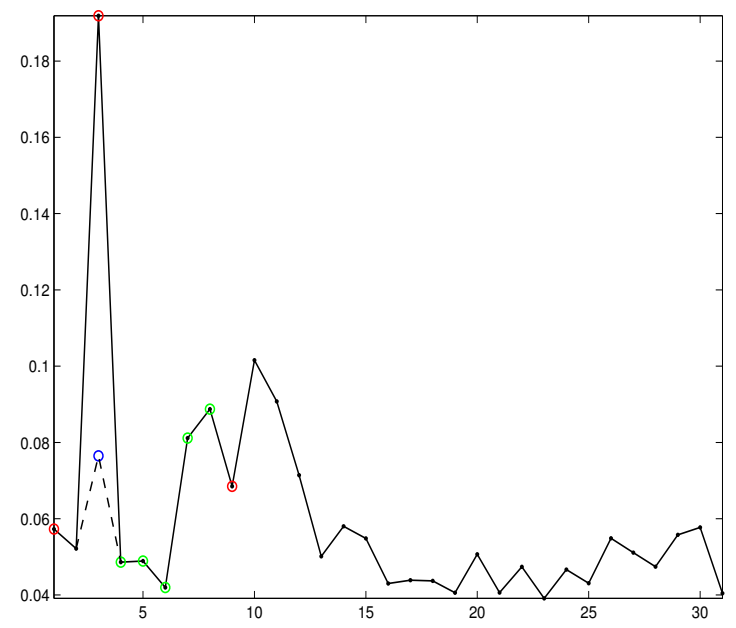

Figure 8. Test 3: NDM curve. Red circle - only one frame in the pair has bleeding. Green circle - both frames in the pair have bleeding. Black dot - none of the frames in the pair has bleeding. The blue circle for pair number 3 is the value of $N D M$ with the modified frame 4 (exhibited in Figure 7) that does not contain bleeding.

larger and this is due not only to the existence of bleeding but also to the fact the content of the images also changes.

\section{Conclusions}

A dissimilarity measure, defined by means of a particular image registration method, aiming at quantifying the dissimilarity of the contents of two consecutive frames in a WCE video, has been used in this paper, to analyse whether it is also able to identify frames with bleeding in the small bowel. The results of the synthetic and semi-synthetic experiments with real WCE images, clearly show that the peaks of the curve representing the dissimilarity measure, identify the frames with bleeding. So, this is a very promising result, that foresees the use of this curve for detecting, easily and quickly, frames with abnormalities in WCE videos. In effect the peaks in this curve (higher or lower peaks) are always generated by one of the following two possibilities:

- The two consecutive frames are very similar but just one of them displays an abnormality. This is clearly the case exemplified in the synthetic and semi-synthetic experiments and it also occurs in real-life WCE images (see Figures 7 and 8). 
- The two consecutive frames exhibit a great difference in their imaged contents, independently of the existence, or not, of an abnormality in at least one frame.

Thus this curve is a graphical tool that quantifies the degree of dissimilarity between consecutive frames in a WCE video and provides the possibility, to a medical doctor, of quickly identifying a set of critical frames, that correspond to the peaks in the curve.

Furthermore, as observed in [10], this dissimilarity measure is also an indicator of the speed of the capsule, since low values mean that the scene does not change (so the capsule is almost still or moves very slowly), while high values mean the scene changes (so the capsule either rotates or moves forward due to peristalis).

Finally, we remark that the experiments described herein for identifying frames with bleeding can be replicated, with appropriate adjustments, for finding frames with other types of abnormalities, as for instance polyps.

\section{References}

[1] D. G. Adler and C. J. Gostout. Wireless capsule endoscopy. Hospital Physician, 39(5):14-22, 2003.

[2] Y. Bao, G.and Ye, U. Khan, X. Zheng, and K. Pahlavan. Modeling of the movement of the endoscopy capsule inside GI tract based on the captured endoscopic images. In International Conference on Modeling, Simulation and Visualization Methods, Las Vegas, 2012.

[3] C. Broit. Optimal registration of deformed images. Ph.D. Thesis, Computer and Information Science, University of Pensylvania, 1981.

[4] G. Ciuti, A. Menciassi, and P. Dario. Capsule endoscopy: from current achievements to open challenges. Biomedical Engineering, IEEE Reviews in, 4:59-72, 2011.

[5] M. Coimbra and J.P.S. Cunha. MPEG-7 visual descriptors-contributions for automated feature extraction in capsule endoscopy. IEEE Transactions on Circuits and Systems for Video Technology, 16:628-637, 2006.

[6] J.P.S. Cunha, M. Coimbra, P. Campos, and J.M. Soares. Automated topographic segmentation and transit time estimation in endoscopic capsule exams. IEEE Transactions on Medical Imaging, 27(1):19-27, 2008.

[7] Pierre-Nicolas D'Halluin, Michel Delvaux, Marie-Georges Lapalus, Sylvie Sacher-Huvelin, Emmanuel Ben Soussan, Laurent Heyries, Bernard Filoche, Jean-Christophe Saurin, Gerard Gay, and Denis Heresbach. Does the suspected blood indicator improve the detection of bleeding lesions by capsule endoscopy? Gastrointestinal endoscopy, 61(2):243-249, 2005.

[8] R. Eliakim. Video capsule colonoscopy: where will we be in 2015? Gastroenterology, 139(5):1468-1471, 2010.

[9] Isabel N Figueiredo, Sunil Kumar, Carlos Leal, and Pedro N Figueiredo. Computer-assisted bleeding detection in wireless capsule endoscopy images. Computer Methods in Biomechanics and Biomedical Engineering: Imaging \& Visualization, 1(4):198-210, 2013. 
[10] Isabel N. Figueiredo, Carlos Leal, Luis Pinto, Pedro N. Figueiredo, and Richard Tsai. An elastic image registration approach for wireless capsule endoscope localization. unpublished (arXiv:1504.06206 [cs.CV]), 2015.

[11] R.D. Francis. Sensitivity and specificity of the red blood identification (rbis) in video capsule endoscopy. In 3rd INt. Conf. Capsule Endoscopy, Miami, FL, USA, Feb 2004.

[12] G. Idan, G. Meron, A. Glukhovsky, and P. Swain. Wireless capsule endoscopy. Nature, 405:417$417,2000$.

[13] B. Li and M. Q.-H-Meng. Computer-aided detection of bleeding regions for capsule endoscopy images. IEEE Transactions on Biomedical Engineering, 56:1032-1039, 2009.

[14] J. Modersitzki. Numerical methods for image registration. OUP Oxford, 2003.

[15] J. Modersitzki. FAIR: flexible algorithms for image registration, volume 6. SIAM, 2009.

[16] A. Moglia, A. Menciassi, and P. Dario. Recent patents on wireless capsule endoscopy. Recent Patents on Biomedical Engineering, 1(1):24-33, 2008.

[17] T. Nakamura and A. Terano. Capsule endoscopy: past, present, and future. Journal of gastroenterology, 43(2):93-99, 2008.

[18] G. Pan, F. Xu, and J. Chen. A novel algorithm for color similarity measurement and the application for bleeding detection in WCE. I.J. Image, Graphics and Signal Processing, 5:1-7, 2011.

[19] G. Pan, G. Yan, X. Qiu, and J. Cui. Bleeding detection in wireless capsule endoscopy based on probabilistic neural network. J. Med. Syst., 35:1477-1484, 2011.

[20] S. C. Park, H. J. Chun, E. S. Kim, B. Keum, Y. S. Seo, Y. S. Kim, Y. T. Jeen, H. S. Lee, S. H. Um, C. D. Kim, and H. S. Ryu. Sensitivity of the suspected blood indicator: An experimental study. World J. Gastroenterolgy, 18(31):4169-4174, 2012.

[21] J. Schanda. Colorimetry: Understanding the CIE system. Wiley \& Sons, NJ, USA, 2007.

[22] E. Spyrou and D. K. Iakovidis. Video-based measurements for wireless capsule endoscope tracking. Measurement Science and Technology, 25(1):015002, 2014.

[23] P. M. Szczypiński, R. D. Sriram, P. VJ Sriram, and D. N. Reddy. A model of deformable rings for interpretation of wireless capsule endoscopic videos. Medical Image Analysis, 13(2):312$324,2009$.

[24] T. D. Than, G. Alici, H. Zhou, and W. Li. A review of localization systems for robotic endoscopic capsules. IEEE Transactions on Biomedical Engineering, 59(9):2387-2399, 2012.

ISABEL NARRA FigueIREDO

CMuC, Department of Mathematics, University of Coimbra, Portugal.

E-mail address: isabelf@mat.uc.pt

$U R L:$ http://www.mat.uc.pt/ isabelf/

Carlos Leal

CMuC, Department of Mathematics, University of Coimbra, Portugal

E-mail address: carlosl@mat.uc.pt

Luís Pinto

CmuC, Department of Mathematics, University of Coimbra, Portugal.

E-mail address: luisp@mat.uc.pt

Pedro Narra Figueiredo

Department of Gastroenterology-CHUC and Faculty of Medicine-UC University of Coimbra, Portugal. 
E-mail address: pnf11@sapo.pt

RICHARD TSAI

Department of Mathematics and ICES, The University of Texas at Austin, Austin, USA, and KTH Royal Institute of Technology, Sweden.

E-mail address: tsai@kth.se

URL: https://www.ices.utexas.edu/people/160/ 